\title{
Towards an optimum design of wave energy converter arrays through an integrated approach of life cycle performance and operational capacity
}

\author{
Alejandro López-Ruiz ${ }^{\mathrm{a}, *}$, Rafael J. Bergillos ${ }^{\mathrm{b}}$, Juan M. Raffo-Caballero ${ }^{\mathrm{a}}$, \\ Miguel Ortega-Sánchez \\ ${ }^{a}$ Departamento de Ingeniería Aeroespacial y Mecánica de Fluidos, Universidad de \\ Sevilla, Camino de los Descubrimientos s/n, 41092, Sevilla, Spain \\ ${ }^{b}$ Andalusian Institute for Earth System Research, University of Granada, Avda. del \\ Mediterráneo, s/n, 18006, Granada, Spain
}

\section{Abstract}

Over the last few decades, several efforts have been made to develop an alternative and sustainable energy source from wind waves. To achieve financial sustainability of this technology, most of the research has focused on analyzing facilities composed of several wave energy converters (WECs) arrays instead of isolated ones. Although the interaction between devices and its implications on the performance of the facilities have been studied previously, these works considered only certain combinations of sea states, limiting the applicability of the results. This work applies a new methodology based on statistical methods to assess the performance of different WEC array distributions during their entire life-cycle in an efficient way, using downscaling techniques and advanced numerical modeling to propagate the wave climate. The results obtained during the hindcasting life-cycle are used to analyze the

\footnotetext{
*Corresponding author alopez50@us.es
} 
maintenance and operation capabilities of the different alternatives of arrays defined for the WEC facility. The interactions between devices and their efficiency considering the associated impact are also quantified. The assessment of these efficiencies during the complete life-cycle of the devices is highly valuable tool for promoters and coastal managers to evaluate different WEC array alternatives. The entire process was applied to a hypothetical array location in the Gulf of Cádiz (southwestern Spain), where three different array distributions were defined. The results show that the distance between WECs is a key parameter that controls the potential energy production, the efficiency of the facility and the interactions between several devices.

Keywords: Wave energy converters, array layout, performance, maintenance, hindcasting, downscaling

\section{Introduction}

During recent years, the development of non-conventional renewable energy technologies has received increasing attention due to the environmental problems derived from the use of fossil fuels. Among these non-conventional sources, marine energy resources can be stored in the form of thermal, kinetic, chemical and biological energy (Khan et al., 2017). Accordingly, many recent works have focused on these types of sources, especially on hydrokinetic energy extraction. Two main technologies have been developed: (1) the extraction of energy from tidal currents using Tidal Energy Converters (TECs; Pacheco and Ferreira, 2016) and (2) the extraction of energy from waves by wave energy converters (WECs; Taylor, 2010).

WECs generate electricity from the kinetic energy of waves through dif- 
ferent physical processes, such as wave overtopping, wave impact or wave oscillation (López et al., 2013). Depending on their specific characteristics, these devices can be fixed not only at maritime infrastructures but also in nearshore or offshore bathymetric zones and usually have dimensions ranging from tens to hundreds of meters (López et al., 2013). To optimize the economic viability of this wave energy resource, the devices are frequently not installed isolated but in arrays or farms of many units Astariz and Iglesias, 2015). However, these arrays must be carefully designed to avoid inefficient interactions between the devices due to the modifications of the wave field that they may generate (O'Boyle et al. 2017).

Numerous studies have focused on the interactions among nearby devices. Initial works analyzed very simple array geometries forced by unidirectional regular waves (Budal, 1977; Falnes, 1980). Later, research interest was focused on more complex design parameters: Babarit (2010) analyzed the interaction between two WECs and the influence of the separation between them, whereas Cruz et al. (2010) used regular and irregular waves to analyze the interactions in the array layout and the control strategy of the facility. Furthermore, Borgarino et al. (2012) presented a study on the interaction between WECs in arrays of 9 to 25 devices within a yearly scale. de Andrés et al. (2014) addressed the optimum array configurations, in terms of power production, for different wave climates around the globe. Engstrom et al. (2013) and Goteman et al. (2014) analyzed fluctuations in the power availability of different arrays configurations, minimizing the power variance for some wave conditions and array geometries. More recently, Bozzi et al. (2017) assessed the performance of different WEC array geometries along the 
Italian offshore platform using some combinations of real sea states.

Despite the importance to potential investors, these previous studies only considered certain wave conditions without focusing on the life-cycle performance of the arrays. The life-cycle assessments of WECs and WEC arrays were focused on their environmental and economic impacts (Thomson et al., 2011; Uihlein, 2016) at different stages of the facilities (i.e., installation, use and dismantling) and energy productions that were roughly estimated (Hans Chr. et al., 2007) using only mean values of the resource rather than obtaining the complete time series during the life-cycle on a high temporal resolution basis. More exhaustive analyses were performed for energy assessments at large scale areas, based on hindcasted wave climate time series. These analyses were performed for different durations, ranging from a few years (Mediavilla and Sepúlveda, 2016) to more than 60 years (Bromirski and Cayan, 2015). For these analyses, different models such as WAVEWATCH (Besio et al., 2015) or MIKE21 (Carr et al., 2016) were employed. However, these studies do not consider the presence of WECs and their influence on the local wave field. To the authors knowledge, López-Ruiz et al. (2016) was the first study where the energy resource was assessed and forecasted for the complete life-cycle of isolated WECs. More recently, Bailey et al. (2017) presented a stochastic model for predicting WEC array power time series, applicable only for arrays of independent WECs.

Although significant advances have been achieved during the last decade, a unified framework to evaluate the performance and operational capabilities of different alternatives of WEC arrays during their complete life-cycle is still lacking. As described previously, some works analyzed only certain combina- 
tions of wave climates, whereas others were focused only on the performance of the facilities. Furthermore, the operational and maintenance capabilities were obtained for large-scale analyses with low spatial and temporal resolutions and without considering the interaction between WECs and hence the local wave conditions (Guanche et al., 2015; Rinaldi et al., 2017) which critically affects the results. This limits the applicability for decision-makers, who usually must make important investments in a technology where the commercial margins are narrow.

The main objective of this work is to present the first unified methodology for assessing the potential life-cycle performance of different geometrical configurations of WEC arrays using a hindcasting wave climate and statistical tools to reduce the computational costs. The methodology obtains not only the energy production of the arrays during their life-time but also their operational and maintenance capabilities, providing a unified framework to evaluate WEC array alternatives. The efficiency of the alternatives is also obtained in terms of the occupied surface and length of the wave front affected by the WECs. These aspects were not analyzed previously using neither experimental nor numerical methods, neglecting the visual, navigational and environmental impact of the arrays. The methodology presented throughout this paper is the first to allow the assessment of these efficiencies during the complete life-cycle of the devices. It can assist promoters and coastal managers during the evaluation of different WEC array alternatives, allowing a more accurate assessment of both the life-cycle performance and maintenance aspects.

The methodology, which will be described throughout the manuscript, 
can be divided into two main stages: (1) the definition of the WEC array configuration and (2) the evaluation of its performance using a numerical model. Furthermore, cutting-edge statistical methods are applied to obtain the wave energy potential for every individual WEC during its life-cycle with a minimum computational cost. The methodology is used to evaluate 9 different array configurations consisting of 9 WEC units in the southwestern coast of Spain. The results are obtained for a set of 25-year hindcast wave data. The different alternatives are evaluated in terms of not only their energy production but also their environmental impact (i.e., occupied surface) as well as operational and maintenance aspects.

The methodology employed in this work applies not only to any type of WEC array (regardless of the particular device employed) but also to any other renewable energy resource that is dependent on atmospheric forces (i.e., wind energy). It was developed to be applied straightforwardly to real applications, as cutting-edge statistical techniques are used in such a way that they can be directly applied by investors and decision-makers to optimize the design of WEC arrays, reducing the gap between research, development and implementation.

\section{Study site}

The southwestern area of the Gulf of Cádiz is one of the few locations along the southern coast of Spain where it is feasible to install a WEC array due to its wide continental shelf $(30 \mathrm{~km})$, relatively low depths and mild slopes (Ortega-Sánchez et al. 2008), with the shelf-break at approximately $120 \mathrm{~m}$ water depth (Fig. 1a-b). In addition, there is a strong wave energy 
potential in this area (Reikard et al., 2016). These characteristics enhance the feasibility of exploiting the wave energy resource according to the guidelines of the Kyoto protocol and the European Union and Spanish energy politics (Besio and Losada, 2008). In this work, the WEC arrays were placed at the widest part of the inner continental shelf, close to Trafalgar Cape, so that the arrays operate in intermediate depths (approximately $34 \mathrm{~m}$ ) but at a considerable distance from the coast $(\simeq 10 \mathrm{~km})$. This location is feasible in terms of economical exploitation (Iglesias and Carballo, 2014, Abanades et al., 2015), and it minimizes the environmental impact of the facility since it may provoke minimum disturbances on sediment transport both at the study site and at the shore.

The area is a mesotidal and swell-dominated coastal environment. The astronomical tide is semi-diurnal with tidal ranges between $1.2 \mathrm{~m}$ and 3.8 m (Ortega-Sánchez et al., 2008). Wave data from SIMAR point 5034009 indicate that the prevailing incoming wave directions are west and westnorthwest (Fig. 1c). The 50\%, 90\% and 99\% exceedance significant wave heights $\left(H_{s}\right)$ in deep water are $1.1 \mathrm{~m}, 2.2 \mathrm{~m}$ and $4.0 \mathrm{~m}$, respectively. During extreme storms, maximum significant wave heights typically exceed $3.5 \mathrm{~m}$.

\section{Methodology}

The methodology applied to hindcast the performance of different WEC arrays geometries in the study site is described in the following sections. Furthermore, we present the tools used to analyze the results, including the procedure to evaluate the relative impact of the different array layouts. 


\subsection{Wave climate}

The main objective of this work is to evaluate the performance of WEC arrays during their life-cycle, which is usually considered to be 25 years (Margheritini et al., 2009; Alonso et al., 2015). Hence, we gathered a wave dataset spanning 25 years, obtained from the hindcast database of Puertos del Estado (Spanish Ministry of Public Works).

We used hindcast synthetic wave data obtained at 4 different locations (Fig. 1b). They correspond to the nodes of a computational mesh with a resolution of approximately $1^{\circ}$ in which data every $3 \mathrm{~h}$ are available. These wind and wave data were obtained through the High Resolution Limited Area Model (HIRLAM, Cats and Wolters, 1996), and the WAM model (Booij et al., 1999), respectively.

SIMAR points 6018024,6026026 and 6028024 correspond to intermediate depths and were used to calibrate the wave propagation model, as described in Section 3.2. These datasets spanned 11 years from 2005 to 2016. SIMAR 6018024 data were also used to define the orientation of the WEC arrays (Section 3.3.2). By contrast, SIMAR 5034009 corresponds to deep-water data spanning 58 years, from 1958 to 2016. Given its length, this dataset was used to obtain the hindcasting database of sea states to be propagated with the numerical model using downscaling techniques, as well as to obtain the 25-year wave climate series.

In terms of computational effort and efficiency, the use of a 25-year wave climate time series to assess energy resources is a challenging task. The propagation of the complete dataset would involve a vast number of cases in the numerical model, which would limit the applicability of the presented 
methodology. To reduce this computational effort, statistical tools were applied through downscaling techniques previously validated for the Mediterranean coast of southern Spain by Bergillos et al. (2016) and López-Ruiz et al. (2016).

First, a database of representative wave conditions $\left(H_{s}, T_{p}\right.$ and $\left.\theta_{m}\right)$ is generated by applying the downscaling method presented by Camus et al. (2011, 2014) to the SIMAR 5034009 data. This first step synthesizes the total dataset of a deep-water wave climate in a reduced number of sea states (300 in this case) representing mild, mean and extreme wave conditions not equally distributed and accounting for the most likely sea states. Second, this database is propagated using the wave propagation model described in Section 3.2. Through these propagations, nearshore wave parameters are obtained and used to compute the wave power $P$ for every sea state of the database. Finally, with these results, the complete 25-year series of $P$ are calculated for every WEC and array geometry through interpolation. A flow chart describing this process is depicted in Fig. (2).

\subsection{Numerical modeling}

Waves were numerically propagated using the SWAN model, which was

designed to simulate random, short-crested waves in coastal regions Booij et al., 1999). The main processes included in the model are refraction due to bottom and current variations; shoaling, blocking, and reflections due to opposing currents; transmission/blockage through/by obstacles; wind effects; whitecapping; depth-induced wave breaking; bottom friction; and non-linear wave-wave interactions.

Thus, the model is able to simulate the effects of obstacles on the wave 
propagation patterns. These obstacles must have at least one mesh length in any of their dimensions to modify wave properties between adjacent grid points. This limits the minimum refinement for the numerical grids, although in this case the limiting factor was the capability of precisely capturing the effects of the obstacles. The effects on wave propagation are threefold: they reduce the wave height of waves propagating behind or over the obstacle all along its length, it reflects waves that impinge the obstacle, and it diffracts waves around its boundaries (Rusu and Guedes Soares, 2013). In the present work a specular reflection was implemented that implies constant reflection and transmission coefficients along the devices.

The model domain consists of two different grids, as shown in Fig. (1). The first is a coarse curvilinear 163x163-cell grid, with cell sizes of approximately $400 \times 400 \mathrm{~m}$. The second is a nested grid covering the area in which the WEC arrays were placed with $244 \times 244$ cells and cell sizes of about $15 \times 15$ $\mathrm{m}$.

The model was run in its stationary mode and was calibrated considering the following physical processes: refraction, white-capping, depth-induced breaking, nonlinear triad interactions, bottom friction and diffraction. The calibrated parameters and the spectral resolution for the frequency and directional spaces are summarized in Table 1. Similar values were obtained for similar applications in the nearby Bay of Cádiz (Zarzuelo et al., 2015). The calibration was performed comparing the model results with the SIMAR points 6018024, 6026026 and 6028028 data (Fig. 1) for the same locations. The calibration period spanned one month starting on September 1st, 2016, and the correlation coefficients $\left(R^{2}\right)$ obtained were higher than 0.87 proving 
Table 1: Parameters of the numerical model

\begin{tabular}{ccc}
\hline Process & Parameter & Value \\
\hline \hline Spectral resolution for & $\Delta f$ & Log. distribution \\
frequency space & $\mathrm{f}_{\min }$ & $0.03 \mathrm{~Hz}$ \\
\hline Spectral resolution for & $\mathrm{f}_{\max }$ & $1 \mathrm{~Hz}$ \\
directional space & $\Delta \theta$ & $5^{\circ}$ \\
\hline Depth induced & $\theta_{\min }$ & 0 \\
breaking & $\alpha$ & $360^{\circ}$ \\
\hline Nonlinear triad & $\gamma$ & 1 \\
interactions & $\alpha$ & 0.1 \\
\hline Bottom friction & Collins & 2.2 \\
\hline Diffraction & Smoothing coeff. & 0.6 \\
\hline
\end{tabular}

the validity of the defined numerical scheme.

\subsection{WEC array layouts}

\subsubsection{Modeling individual WECs}

This work focuses on floating overtopping WECs, which use a sloping plate that leads the waves to overtop into a reservoir located immediately behind it. The energy is extracted by low head turbines, using the difference in water levels between the reservoir and the average sea water level (Vicinanza et al., 2012). Specifically, we applied the methodology to the 
WaveCat prototype device (Fernandez et al., 2012, Allen et al., 2016). This overtopping WEC has an overall width of $D \simeq 100 \mathrm{~m}$ and is moored in a single point to the seabed, passively orienting itself along the direction of wave propagation (Allen et al., 2016).

The WECs were included in SWAN as obstacles with a circular shape, so that the devices always expose the same width to the incident waves, regardless of the incoming wave direction, simulating the self-orientation behavior of the device. As described in Section 3.2, SWAN requires the definition of reflection and transmission coefficients to account for the effect of the presence of obstacles. These coefficients are partially confidential for commercial reasons, but in the case of the WaveCat WECs, Fernandez et al. (2012) presented values obtained from experimental tests. Although different results were obtained depending on the wave conditions (variations lower than $10 \%$ ), based on the results of these tests, we adopted constant mean values of $k_{t}=0.75$ and $k_{r}=0.44$ for the transmission and reflection coefficients, respectively. Fig. (3) shows six examples of the results obtained using these coefficients for two sea states and three array layouts. The effects of the obstacles on the wave height distribution are clearly observed.

\subsection{2. $W E C$ arrays}

Four variables define the geometrical layouts of WEC arrays: (1) the number of devices, $N,(2)$ the shape of the array, (3) the distance between WECs, $W$, and (4) the orientation of the array. In this paper, arrays of $N=9$ and distances between WECs of $W=2 D, 4 D$ and $6 D$ were defined, according to previous works (Bozzi et al., 2017; Mercadé Ruiz et al., 2017). Furthermore, three different shapes of WEC arrays were considered (Fig. 
4): (1) regular array of $3 \times 3$ elements (Aligned), (2) staggered shape in three columns of 4, 3 and 2 devices, attempting to minimize the effect of the wave trail generated for the outer devices (Staggered), and (3) arrow shape, trying to avoid shadowing effects between WECs (Arrow). Thus, a total of 9 layouts with 9 WECs each were tested.

The arrays were oriented to the main incoming wave direction (in terms of wave energy). To obtain this direction, the wave energy resource $P$ was obtained for the SIMAR point 6018024, which is the nearest to the location of the arrays (Fig. 1). The assessment of $P$ is described in Section 3.4 . and the results are shown in Fig. (5). They reveal that the majority of the wave energy is provided by westerly waves. Hence, the three geometries were oriented to the west, as depicted in Fig. (4).

\subsection{Assessment of the wave energy resource}

The available wave energy resource was evaluated in terms of wave power per unit of wave crest length $(P$, in $\mathrm{W} / \mathrm{m})$. This was obtained from the spectral output of the wave propagation model as:

$$
P=\rho g \int_{0}^{2 \pi} \int_{0}^{\infty} S(f, \theta) c_{g}(f, h) d f d \theta
$$

where $\rho$ is the water density, $g$ is the acceleration of gravity, $S(f, \theta)$ is the directional wave spectrum, $f$ is the frequency, $\theta$ is the propagation direction of the spectral component, $c_{g}$ is the group celerity and $h$ is the water depth. Eq. (1) can be approximated by (Besio et al., 2015):

$$
P=\frac{1}{16} \rho g H_{m 0}^{2} c_{g}
$$


where $H_{m 0}$ is the spectral wave height evaluated from the wave energy spectrum, whose spectral moments are defined as:

$$
m_{i}=\int_{0}^{2 \pi} \int_{0}^{\infty} S(f, \theta) f^{i} d f d \theta
$$

Thus, $H_{m 0}=4 \sqrt{m_{0}}$ and the wave period used to obtain $c_{g}$ is $T_{m-1,0}=$ $m_{-1} / m_{0}$ (Veigas et al., 2014; Veigas and Iglesias, 2014; Besio et al., 2015).

\subsection{Analysis of the results}

Four main aspects of the WEC arrays were analyzed: (1) the performance of the different geometrical layouts in terms of potential energy production, (2) the interaction between WECs, which is positive (negative) if there is a power production gain (loss) compared with isolated systems, (3) the relative performance of the arrays, considering their impact in terms of the occupied surface and the maximum length in which the waves are perturbed, and (4) the maintenance and operational possibilities. For this purpose, 25 years of $P$ were interpolated using the deep-water wave data of SIMAR 5034009 from January 1st, 1992, to December 31st, 2016.

For the first aspect, the average, $95 \%$ percentile and standard deviation of $P$ were obtained based on the hindcast data. For the second aspect, two different approaches were followed to quantify the interactions: (1) assessment of the root mean square error (RMSE) and the correlation coefficient $(R)$ between the individual performance of the WECs in an array and the perfor-

mance of an isolated WEC and (2) assessment of the $q$-factor. RMSE and $R$ are used to analyze the interaction because the main disturbances between the $P$ obtained for different devices in an array are due to the disturbances 
in the wave field provoked by the WECs, given the regular bathymetry and constant depth in the study zone. Regarding the $q$-factor, it is defined as the ratio between the power output of an array of $N$ WECs and the power output of $N$ isolated WECs (Bozzi et al., 2017):

$$
q=\frac{P_{\text {array }}}{N * P_{\text {isoWEC }}}
$$

where $P_{\text {isowec }}$ is the energy resource for an isolated WEC located at the geometrical center of the array (Fig. 4). This was adopted because depth variations across the zone are lower than $10 \%$, so that $P$ variations between isolated WECs at different places inside the zone are negligible.

For the third aspect, two parameters were used to quantify the impact: the surface occupied by the facility $\left(S_{f}\right)$, defined as the area inside the envelope of the WECs (Fig. 4), and the maximum width of the facility that opposes incoming waves $\left(W_{f}\right)$. With these parameters, the $P$ per unit of $S_{f}\left(P_{S}\right)$ and $W_{f}\left(P_{W}\right)$ were assessed to analyze the efficiency of each array configuration in terms of environmental and visual impacts.

In the case of the operation and maintenance of the WECs, a similar approach to that presented by López-Ruiz et al. (2016) was adopted, analyzing four different parameters. The first is the availability, defined as the percentage of time in which the WECs can produce electricity with local wave conditions, considering the range of operational wave conditions of the particular devices installed. The second is the accessibility, which is the percentage of time in which the device may be accessed for maintenance tasks. This factor depends on the meteorological and wave conditions and the type of WEC and vessels used (Guanche et al. 2014). The third parameter is the 
mean monthly number of weather windows (wws) of a certain duration, which are necessary to repair and maintain the WECs. These intervals include the travel time, which shortens the effective work time. Last, we computed the waiting period (wp), which is defined as the time interval between wws of a certain duration. Therefore, this factor accounts for the mean time that the maintenance workers must wait until the weather conditions are suitable for a repair activity of a certain duration.

\section{Results}

\subsection{Energetics performance of the array layouts}

The mean, 95th percentile and standard deviation of $P$ for the 9 array layouts are shown in Fig. (6). The three curves show similar trends: for the same array shape, $P$ increases with an increasing distance between arrays. Variations between layouts of the same shape are more evident for the Aligned and Staggered arrays, with differences up to $20 \%$ for the mean $P$ in the case of the Aligned array. Differences between layouts of the same shape are much smaller for the Arrow array, with variations between $W=4 D$ and $W=6 D$ being almost negligible $(<1 \%)$. These results highlight that the performance is lower as the distance between WECs decreases. Moreover, the performance of the Arrow is significantly higher than the other two geometries due to the lower shadowing effects between WECs (Fig. 6).

Fig. (7) depicts the mean $P$ for the 25-year life-cycle for each array. The tendencies are again very similar for the Aligned (Fig. 7a-c) and Staggered (Fig. 7d-f) shapes: $P$ is higher for the WECs located in the western column due to their higher exposure to more energetic sea states (Fig. 5). The mean 
$P$ decreases to the east with no significant differences between WECs located in the same column.

For the Arrow shape, the results indicate that the north wing of the Arrow is potentially capable of extracting more energy from waves, with higher values of $P$. This is attributed to the sheltering effect that WECs on the south wing have on themselves under WNW and SE waves, which are of certain importance according to Fig. (5). In contrast, WECs of the north wing do not interact between themselves. In any case, the differences between WECs for this shape are much lower than those for the other two geometries, as shown in Fig. (8). It is observed that the variability decreases with increasing distance between WECs, indicating that the interactions between WECs are less important. The variability of the Arrow layouts is considerably lower than in the other geometries.

\subsection{Analysis of the interactions between WECs}

To quantify the interactions between WECs, the root mean square error (RMSE) and the correlation coefficient (R) for the mean $P$ between WECs in the array and an isolated WEC in the geometrical center of the array were obtained. The results indicate that the differences between the WECs in the arrays and the isolated WEC are clearly greater for the Aligned and the Staggered shapes (Fig. 9), highlighting that the interactions between WECs are more important for these layouts. In the case of the Aligned geometry, WECs located at the west side of the layout present the lowest differences between the array and the isolated cases due to the predominance of $\mathrm{W}$ and SW waves. For the Staggered geometry, the correlations are improved with respect to the previous shape, as the west column of the WECs (1 to 4) has 
one more device and because the Staggered location of WECs in the central column ( 5 to 7 ) avoids the interaction with those of the west column. In the case of the Arrow geometry, $R$ is below 0.997 only for WECs 1 to 4 in the $2 D$ layout. These WECs on the north wing of the Arrow are clearly affected by the prevailing direction of the most energetic waves (WSW sector, Fig. 5). The results show that a separation $W \geq 4 D$ is enough to minimize these interactions. Finally, the RMSE decreases as $W$ increases, whereas $R$ increases with $W$, except for the WEC number 9 in the Arrow 6D layout.

RMSE and $R$ provide insights into the differences between the values of $P$ obtained for the arrays and those for an isolated WEC. However, they do not identify whether these differences lead to an increase in energy production compared to isolated WECs. To quantify this aspect, the $q-$ factor was obtained for every array layout. Fig. (10) shows the statistical results for this factor obtained for each array layout at each WEC location. The $q$-factor is lower than 1 except for certain WECs in the case of the Staggered geometry, i.e., the interference between WECs is generally negative (an array of 9 WECs produces less energy than 9 isolated WECs). Moreover, the closer the WECs, the lower the $q$-factor, except for the case of the Arrow 6D layout. The variability of the $q$-factor for the same array layout also reduces as the distance between WECs decreases.

Fig. (11) depicts the mean $q$-factor obtained for the 25-year life cycle of the WECs. Differences between the Arrow geometry and the other two are evident: whereas the minimum values are approximately 0.85 in the case of the Arrow layouts, they descend to 0.55 for the other two geometries for $W=2 D$, with differences up to $40 \%$ between the WECs less and more 
influenced by the interactions. Positive interactions $(q>1)$ between WECs are found for one WEC and one value of $W$ in the Aligned and Staggered geometries, whereas they are found for two WECs (3 and 4) in the case of the Arrow geometry. Regarding the distribution of the $q$-factor along the arrays, WECs located in the west column for Aligned and Staggered geometries are those with the highest $q$-factor, especially the WEC located more to the south. In the case of the Arrow geometry, the highest $q$-factor values were obtained for the north wing of the layouts.

\subsection{Effectiveness of the array layouts}

Fig. (12) shows the effectiveness of the different array distributions, considering the energy production per unit of the occupied surface and facility width. This effectiveness is quantified as the non-dimensional assessment of the energy resource to facilitate the comparison between array layouts. The results reveal that the percent variations in the effectiveness are significantly greater than those in the mean energy resource. As an example, the array layout with the minimum mean energy is only $25 \%$ lower than the one with the maximum value, whereas the minimum effectivenesses are $83 \%$ and $92 \%$ lower than the maximum values of $P_{W}$ and $P_{S}$, respectively. The reduction of the effectiveness from $W=2 D$ to $W=4 D$ is greater than from $W=4 D$ to $W=6 D$, as exhibited by the slopes of the green and red lines in Fig. (12). 


\subsection{Operational and maintenance aspects}

\subsubsection{Availability}

The WECs are able to produce energy if the wave height at the entrance of the WEC ranges between the minimum and maximum thresholds, which depend on the mechanical characteristics of the devices. In the case of the WaveCat WECs, there is no available information regarding this topic due to their early stage of development, and therefore, typical values for other types of WECs $\left(H_{s} \in[0.75,5.9]\right)$ were used (Guanche et al., 2014: López-Ruiz et al., 2016).

The results depicted in Fig. (13) indicate that availability increases with higher distances between WECs, except for the case of the Arrow geometry, in which the higher availability is obtained for the intermediate $W$. For this $W$, the shadowed areas behind the more exposed WECs corresponded to the areas between the WECs of the other line of the arrow, minimizing the interactions. Moreover, lower values are obtained for the WEC with a lower energy performance, highlighting that the limiting factor for the availability is the lower threshold, i.e., the occurrence of low wave heights. Similar results were found for the Aligned and Staggered geometries, although contrary to the analysis of energy performance of the arrays; in this case, the worst layout in terms of availability is the Staggered with $W=2 D$, where there are 4 WECs with an availability lower than $40 \%$.

By contrast, the Arrow geometry presents higher availabilities and a lower variability between devices, with values over $53 \%$ for every WEC and differences below $10 \%$ between the maximum and minimum availabilities in each layout. The maximum availability is almost equal for the 9 layouts defined. 
These maximum values correspond to the WECs less influenced by the presence of the other devices. Hence, the best geometry in terms of availability is the Arrow, in particular the array with $W=4 D$, for which the WECs are producing energy a large portion of the time.

\subsubsection{Accessibility}

In the case of the Gulf of Cádiz, considering the typical wave conditions and the vessels usually employed in the zone, a threshold of $H=1.5 \mathrm{~m}$ was used (Guanche et al., 2014). Although other variables such as the wind speed are also relevant for the accessibility, the wave height is the most limiting factor for the operationality of the vessels (López-Ruiz et al., 2016). Thus, this variable was adopted as the single variable defining accessibility.

The results show that the WECs with the lower accessibility are those for which the interactions between devices are less important (Fig. 14). In the case of the Aligned and Staggered geometries, they correspond to the west column of devices (1, 4, 7 and 1 to 4 , respectively), presenting accessibilities of 82-85\%. The major differences between WECs for these geometries are found for the layouts with $W=2 D$. Values up to $94 \%$ were obtained for the devices located northeast of the arrays, influenced by the sheltering effect of the other devices. The variability in the accessibility to the devices between WECs of the same layout is much lower for the Arrow geometry. Furthermore, median values of the WECs (red lines in the lower panel of Fig. (14) are lower than $83 \%$. 


\subsubsection{Number of weather windows}

For the mean monthly number of weather windows (wws hereinafter), periods of 6, 12 and $24 \mathrm{~h}$ were considered. As in Guanche et al. (2014) and López-Ruiz et al. (2016), the number of consecutive periods of $\mathrm{x}$ hours wws has been considered. Thus, if there is a period of $48 \mathrm{~h}$ with accessible weather conditions, 8 wws of $6 \mathrm{~h}, 4$ wws of $12 \mathrm{~h}$ and 4 wws of $24 \mathrm{~h}$ are obtained. During these periods, wave heights under $1.5 \mathrm{~m}$ were calculated in the vicinity of the devices, and a maintenance vessel would be able to approach the WECs to perform repair or inspection tasks. However, because the vessels must travel to the respective devices, the wave conditions surrounding the facility must also be considered. Consequently, the wave heights obtained for a scenario without arrays were used. Nevertheless, the possible trails of higher wave heights (see Fig. 3 for instance) were not considered because this would require a special analysis of the vessel trajectories that is beyond the scope of this work.

Results are shown in Fig. (15). The patterns in the number of wws are almost equal for the three periods considered, and the variations between WECs of different layouts are under $1 \%$. Therefore, the limiting factor for the wws are the wave conditions during travel to the facility instead of the conditions while approaching the individual devices. For wws of $6 \mathrm{~h}$, considering a mean number of wws of 97.1 , approximately $83 \%$ of the possible wws are available to reach the devices, since a full mean month accessibility corresponds to 120 wws. This proportion reduces to $79 \%$ and $76 \%$ for wws of 12 and $24 \mathrm{~h}$, respectively. 


\subsubsection{Waiting period}

Periods of inaccessibility were also analyzed by calculating the waiting period (wp hereinafter) between wws. If a failure is detected in one or more devices and a maintenance task is required, it will only occur once the maintenance workers have an available ww to reach the WECs. In this section, the definition of the wws is the same as in the previous one. Thus, conditions for both travel to the facility and approach to the WECs are considered.

Fig. (16) shows that the results are very similar to those of the number of wws. The average wp for wws of $6 \mathrm{~h}$ is approximately $7.48 \mathrm{~h}$, whereas it increases up to 15.3 and $31.9 \mathrm{~h}$ for wws of 12 and $24 \mathrm{~h}$, respectively. The variability between WECs is again under 1\%, indicating that the wave conditions during travel to the facility are the limiting factor.

\section{Conclusions and final remarks}

This paper assesses the performance of different geometrical configurations of WEC arrays during their entire life-cycle, using statistical tools and an advanced wave propagation model to reduce the computational costs. The maintenance and operation capabilities, as well as the interactions between devices, were also quantified. For that, three geometries of 9-WEC arrays (Aligned, Staggered and Arrow) with three different spacings between WECs in the Gulf of Cádiz (southwestern Spain) were tested. After the analysis of the results, the following conclusions were drawn:

- The Arrow shape is the most efficient, with improvements in the mean energy produced during the life-cycle up to $20 \%$ compared to the other geometries. For a given geometry, the closer the WECs, the lower the 
total energy and the higher the variability between the energy extracted by the individual WECs.

- The interaction between WECs reduces the available resource up to 40\% for the Aligned and Staggered geometries, whereas the interaction is less significant for the Arrow configuration, increasing the total energy resource.

- The Aligned and Staggered geometries are the most efficient layouts, evaluated as the potential energy per unit of occupied surface and per unit of maximum width of the facility. The efficiency decreases with increasing distances. For the Arrow geometry, the efficiencies are up to $90 \%$ lower compared to the other geometries.

- The Arrow is the best alternative in terms of maintenance and operation, since it presents the highest availabilities and similar number of weather windows and waiting periods than the remaining layouts. As in the cases of energy performance and efficiency, the increase in the distance between devices also undermines maintenace and operation tasks.

The major achievement of this work is the development of a new methodology to efficiently design the shape, orientation and distance between WECs in terms of: (1) maximizing the performance and efficiency of the array and (2) minimizing the interactions between WECs and total occupied surface. The methodology can be straightforwardly applied to any WEC array, regardless of the type and number of devices employed or the shape or the 
location of the facility. It can also be applied to evaluate design alternatives for other renewable energy technologies dependent on meteorological agents.

\section{Acknowledgments}

This research was supported by the research group TEP-209 (Junta de Andalucía, gdfa.ugr.es). The work of the second author was funded by the Spanish Ministry of Economy and Competitiveness (Research Contract BES2013-062617). The work of the third author was funded by the University of

Seville (Initiation on Research Grant). Two anonymous reviewers and the Editors of the Journal are acknowledged for their comments and suggestions which improved significantly the manuscript.

\section{References}

Abanades, J., Greaves, D., Iglesias, G., 2015. Coastal defence using wave farms: The role of farm-to-coast distance. Renewable Energy 75, 572-582. doi:10.1016/j.renene.2014.10.048.

Allen, J., Sampanis, K., Wan, J., Greaves, D., Miles, J., Iglesias, G., 2016. Laboratory Tests in the Development of WaveCat. Sustainability 8. doi:10. $3390 /$ su8121339.

Alonso, R., Solari, S., Teixeira, L., 2015. Wave energy resource assessment in Uruguay. Energy 93, 683-696. doi:10.1016/j.energy.2015.08.114.

de Andrés, A., Guanche, R., Meneses, L., Vidal, C., Losada, I., 2014. Factors that influence array layout on wave energy farms. Ocean Engineering 82, 32 - 41. doi:10.1016/j.oceaneng. 2014.02.027. 
Astariz, S., Iglesias, G., 2015. The economics of wave energy: A review. Renewable and Sustainable Energy Reviews 45, 397-408. doi:10.1016/j . rser.2015.01.061.

Babarit, A., 2010. Impact of long separating distances on the energy production of two interacting wave energy converters. Ocean Engineering 37, 718 - 729. doi:10.1016/j.oceaneng.2010.02.002.

Bailey, H., Robertson, B., Buckham, B., 2017. Variability and Stochastic Simulation of Power from Wave Energy Converter Arrays. Renewable Energy doi:10.1016/j.renene.2017.08.052.

Bergillos, R., López-Ruiz, A., Ortega-Sánchez, M., Masselink, G., Losada, M., 2016. Implications of delta retreat on wave propagation and longshore sediment transport - Guadalfeo case study (southern Spain). Marine Geology 382, 1-16.

Besio, G., Losada, M.A., 2008. Sediment transport patterns at Trafalgar offshore windfarm. Ocean Engineering 35, 653-665. doi:10.1016/ j.oceaneng. 2008.01 .002 .

Besio, G., Mentaschi, L., Massino, A., 2015. Wave energy resource assessment in the Mediterranean Sea on the basis of a 35-year hindcast. Energy 94, 50-63. doi:10.1016/j.energy.2015.10.044.

Booij, N., Ris, R.C., Holthuijsen, L.H., 1999. A third-generation wave model for coastal regions 1. Model description and validation. Journal of Geophysical Research 104, 7649-7666. doi:10.1029/98JC02622. 
Borgarino, B., Babarit, A., Ferrant, P., 2012. Impact of wave interactions effects on energy absorption in large arrays of wave energy converters. Ocean Engineering 41, 79 - 88. doi:10.1016/j.oceaneng.2011.12.025.

Bozzi, S., Giassi, M., Moreno Miquel, A., Antonini, A., Bizzozero, F., Gruosso, G., Archetti, R., Passoni, G., 2017. Wave energy farm design in real wave climates: the Italian offshore. Energy 122, 378-389. doi:10.1016/j.energy.2017.01.094.

Bromirski, P.D., Cayan, D.R., 2015. Wave power variability and trends across the north atlantic influenced by decadal climate patterns. Journal of Geophysical Research: Oceans 120, 3419-3443. doi 10.1002/2014JC010440.

Budal, K., 1977. Theory for absorption of wave power by a system of interacting bodies. Journal of Ship Research 21, 248-253.

Camus, P., Mendez, F.J., Medina, R., 2011. A hybrid efficient method to downscale wave climate to coastal areas. Coastal Engineering 58, 851-862.

Camus, P., Menéndez, M., Méndez, F.J., Izaguirre, C., Espejo, A., Cánovas, V., Pérez, J., Rueda, A., Losada, I.J., Medina, R., 2014. A weathertype statistical downscaling framework for ocean wave climate. Journal of Geophysical Research C: Oceans 119, 7389-7405.

Carr, D., Gill, L., McNabola, A., 2016. Development of a high resolution wave climate modelling methodology for offshore, nearshore and onshore locations of interest. International Journal of Marine Energy 16, 30-40. doi:10.1016/j.ijome.2016.05.007. 
Cats, G., Wolters, L., 1996. The HIRLAM project. IEEE Computational Science and Engineering 3, 4-7.

Cruz, J., Sykes, R., Siddorn, P., Taylor, R., 2010. Estimating the loads and energy yield of arrays of wave energy converters under realistic seas. IET Renewable Power Generation 4, 488-497. doi:10.1049/iet-rpg.2009. 0174. cited By 20.

Engstrom, J., Eriksson, M., Goteman, M., Isberg, J., Leijon, M., 2013. Performance of large arrays of point absorbing direct-driven wave energy converters. Journal of Applied Physics 114, 204502. doi:10.1063/1.4833241.

Falnes, J., 1980. Radiation impedance matrix and optimum power absorption for interacting oscillators in surface waves. Applied Ocean Research 2, 75 - 80. doi:10.1016/0141-1187(80)90032-2.

Fernandez, H., Iglesias, G., Carballo, R., Castro, A., Fraguela, J., TaveiraPinto, F., Sanchez, M., 2012. The new wave energy converter wavecat: Concept and laboratory tests. Marine Structures 29, 58 - 70. doi:10. 1016/j.marstruc.2012.10.002.

Goteman, M., Engstrom, J., Eriksson, M., Isberg, J., Leijon, M., 2014. Methods of reducing power fluctuations in wave energy parks. Journal of Renewable and Sustainable Energy 6, 043103. doi:10.1063/1.4889880.

Guanche, R., de Andrés, A.D., Simal, P.D., Vidal, C., Losada, I.J., 2014. Uncertainty analysis of wave energy farms financial indicators. Renewable Energy 68, 570-580. doi:10.1016/j.renene.2014.02.046. 
Guanche, R., De Andrés, A., Losada, I.J., Vidal, C., 2015. A global analysis of the operation and maintenance role on the placing of wave energy farms. Energy Conversion and Management 106, 440-456. doi:10.1016/ j.enconman.2015.09.022.

Hans Chr., S., Stefan, N., Stefan, A., Hauschild, M., 2007. Life cycle assessment of the wave energy converter: Wave dragon.

Iglesias, G., Carballo, R., 2014. Wave farm impact: The role of farm-to-coast distance. Renewable Energy 69, 375-385. doi:10.1016/j.renene.2014. 03.059 .

Khan, N., Kalair, A., Abas, N., Haider, A., 2017. Review of ocean tidal, wave and thermal energy technologies. Renewable and Sustainable Energy Reviews 72, 590-604. doi:10.1016/j.rser.2017.01.079.

López, I., Andreu, J., Ceballos, S., Martínez De Alegría, I., Kortabarria, I., 2013. Review of wave energy technologies and the necessary powerequipment. Renewable and Sustainable Energy Reviews 27, 413-434. doi:10.1016/j.rser.2013.07.009.

López-Ruiz, A., Bergillos, R.J., Ortega-Sánchez, M., 2016. The importance of wave climate forecasting on the decision-making process for nearshore wave energy exploitation. Applied Energy 182, 191-203. doi:10.1016/j. apenergy.2016.08.088.

Margheritini, L., Vicinanza, D., Frigaard, P., 2009. SSG wave energy converter: Design, reliability and hydraulic performance of an innovative over- 
topping device. Renewable Energy 34, 1371-1380. doi:10.1016/j.renene. 2008.09 .009 .

Mediavilla, D.G., Sepúlveda, H.H., 2016. Nearshore assessment of wave energy resources in central Chile (2009-2010). Renewable Energy 90, 136-144. doi:10.1016/j.renene.2015.12.066.

Mercadé Ruiz, P., Ferri, F., Kofoed, J., 2017. Experimental Validation of a Wave Energy Converter Array Hydrodynamics Tool. Sustainability 9, 115. doi:10.3390/su9010115.

O’Boyle, L., Elsäßer, B., Whittaker, T., 2017. Experimental Measurement of Wave Field Variations around Wave Energy Converter Arrays. Sustainability 9, 70. doi:10.3390/su9010070.

Ortega-Sánchez, M., Fachin, S., Sancho, F., Losada, M.a., 2008. Relation between beachface morphology and wave climate at Trafalgar beach (Cádiz, Spain). Geomorphology 99, 171-185. doi:10.1016/j.geomorph.2007.10. 013 .

Pacheco, A., Ferreira, 2016. Hydrodynamic changes imposed by tidal energy converters on extracting energy on a real case scenario. Applied Energy 180, 369-385. doi:10.1016/j.apenergy.2016.07.132.

Reikard, G., Robertson, B., Bidlot, J., 2016. Wave Energy Worldwide: Simulating wave farms, forecasting, and calculating reserves. International Journal of Marine Energy In Review, 156-185. doi:10.1016/j.ijome. 2017.01 .004 . 
Rinaldi, G., Thies, P., Walker, R., Johanning, L., 2017. A decision support model to optimise the operation and maintenance strategies of an offshore renewable energy farm. Ocean Engineering 145, 250-262. doi:10.1016/j. oceaneng.2017.08.019.

Rusu, E., Guedes Soares, C., 2013. Coastal impact induced by a Pelamis wave farm operating in the Portuguese nearshore. Renewable Energy 58, 34-49. doi:10.1016/j.renene.2013.03.001.

Taylor, R., 2010. Non-conventional energy sources. International Journal of Earth Sciences and Engineering 3, 125-141. Cited By 0.

Thomson, R.C., Harrison, G.P., Chick, J.P., 2011. Full life cycle assessment of a wave energy converter, in: IET Conference on Renewable Power Generation (RPG 2011), pp. 1-6. doi:10.1049/cp.2011.0124.

Uihlein, A., 2016. Life cycle assessment of ocean energy technologies. International Journal of Life Cycle Assessment 21, 1425-1437. doi:10.1007/ s11367-016-1120-y.

Veigas, M., Iglesias, G., 2014. Potentials of a hybrid offshore farm for the island of Fuerteventura. Energy Conversion and Management 86, 300-308. doi:10.1016/j.enconman.2014.05.032.

Veigas, M., López, M., Iglesias, G., 2014. Assessing the optimal location for a shoreline wave energy converter. Applied Energy 132, 404-411. doi:10. 1016/j.apenergy.2014.07.067.

Vicinanza, D., Margheritini, L., Kofoed, J.P., Buccino, M., 2012. The SSG 


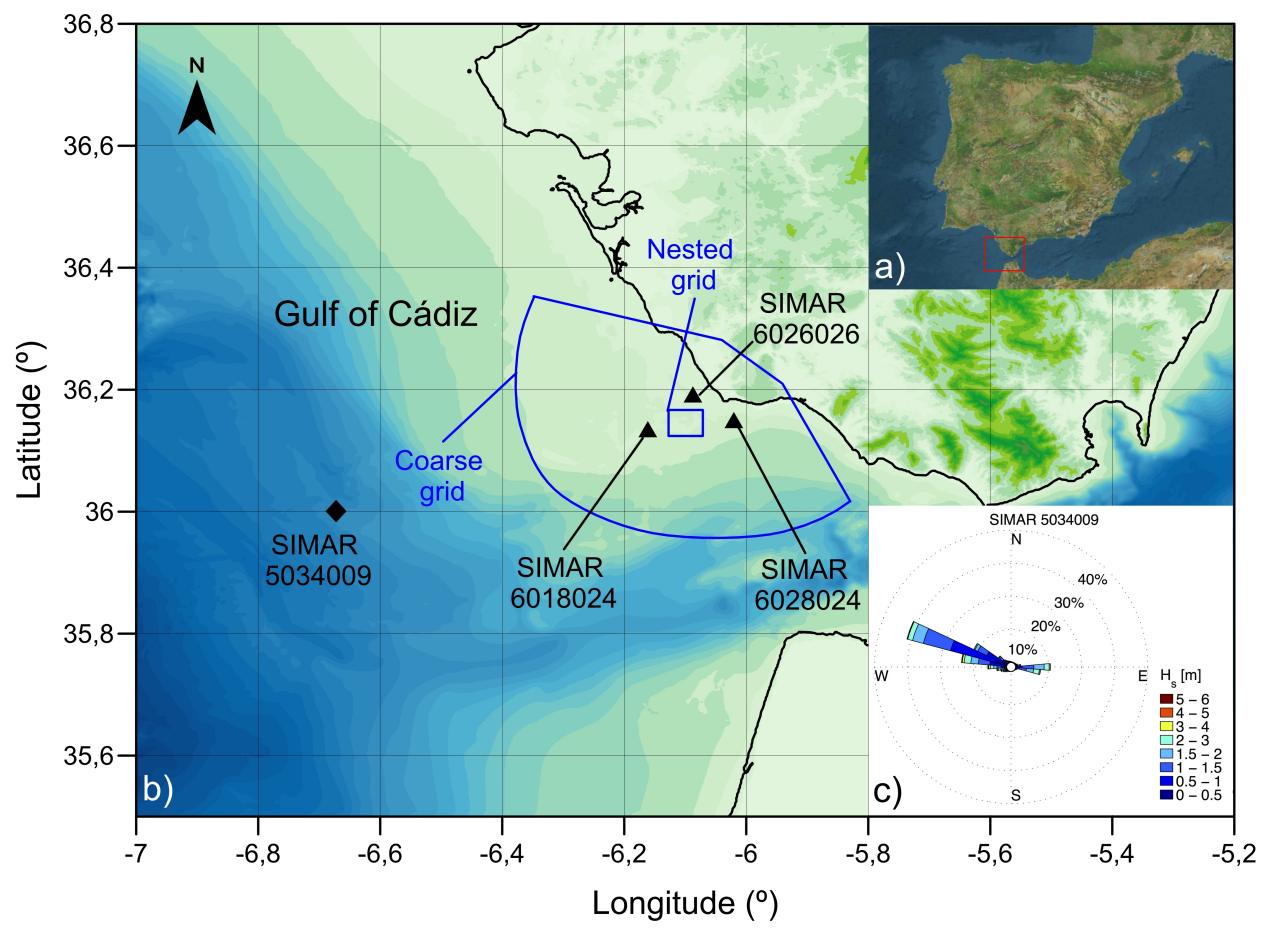

Figure 1: a) Location of the study site (Gulf of Cádiz, southwestern Spain); b) delimitation of the area where the WEC arrays were placed (small blue polygon), boundaries of the grids used in the numerical model (blue polygons), and location of the point in which wave data were available (black squares and triangles); c) wave rose based on the SIMAR 5034009 data.

wave energy converter: Performance, status and recent developments. Energies 5, 193-226. doi:10.3390/en5020193.

Zarzuelo, C., Díez-minguito, M., Ortega-Sánchez, M., López-Ruiz, A., Losada, M.A., 2015. Hydrodynamics response to planned human interventions in a highly altered embayment: The example of the Bay of Cádiz. Estuarine, Coastal and Shelf Science 167, 1-11. doi:10.1016/j.ecss. 2015.07.010. 


\section{Life-cycle performace of WEC arrays}

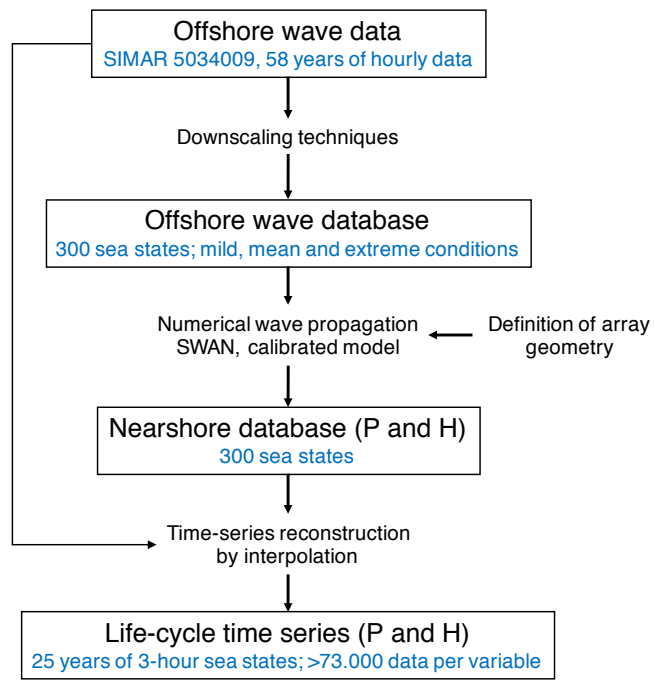

Figure 2: Flow chart of the methodology employed. The text in boxes correspond to the results obtained. The text in blue refers to the particular results obtained at the study site 

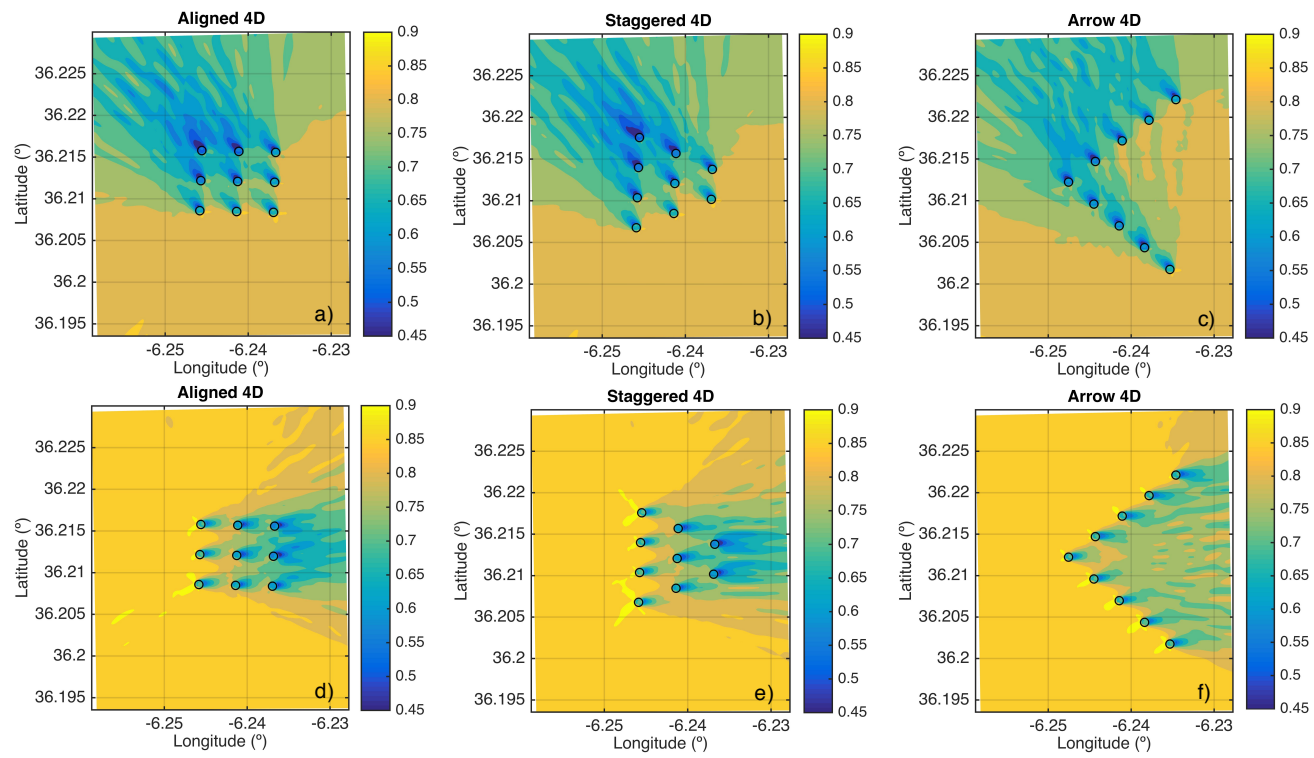

Figure 3: Propagation coefficients (relation between deep-water and local wave heights) for the Aligned (left panels), Staggered (central panels) and Arrow (right panels) geometries under southeasterly (upper panels) and westerly (lower panels) waves with $H=3.4 \mathrm{~m}$ and $H=6.1 \mathrm{~m}$, and $T=10.8 \mathrm{~s}$ and $T=11.3 \mathrm{~s}$, respectively. These sea states correspond to cases 275 and 298 of the defined database.
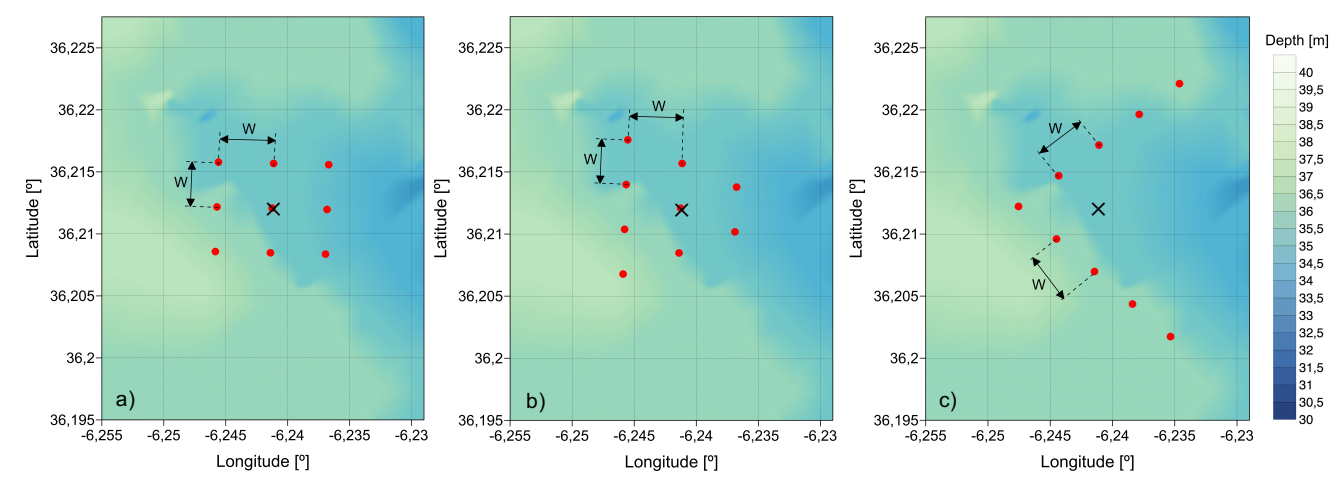

Figure 4: Shape of the WEC arrays defined: a) Aligned, b) Staggered, and c) Arrow. W represents the separation between WECs. The examples depicted correspond to $W=4 D$. The black crosses indicate the geometrical center of the layouts. 

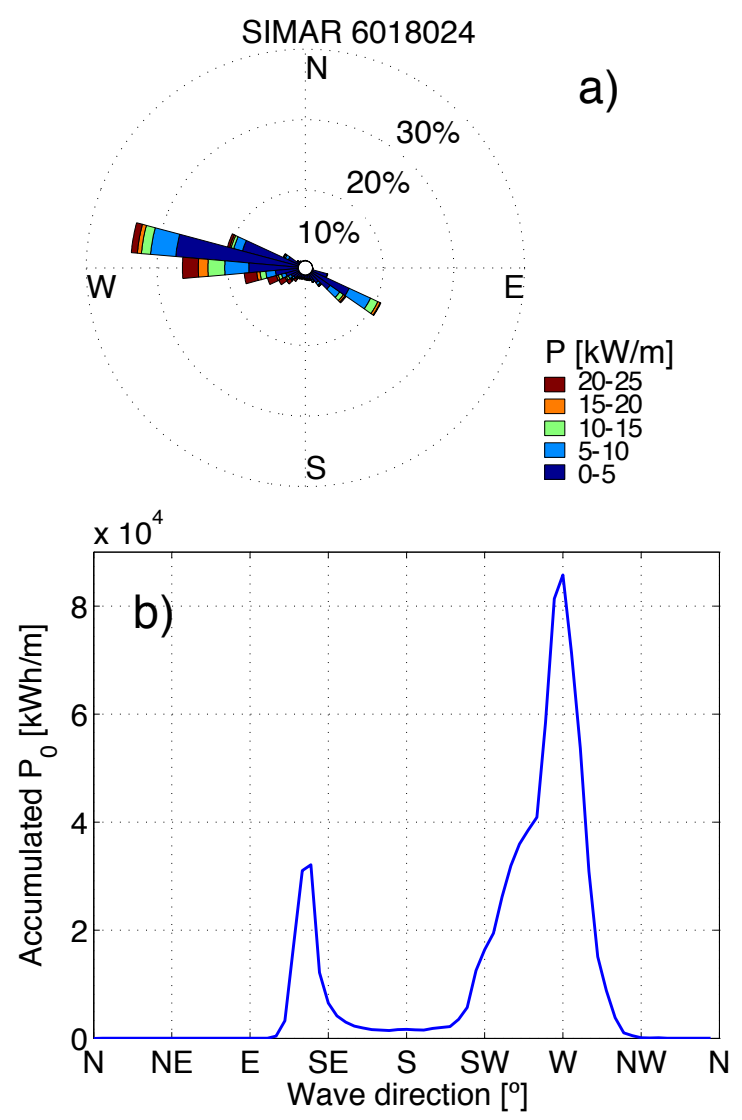

Figure 5: Wave energy resource $(P)$ obtained for SIMAR point 6018024 data: a) $P$ rose, and b) accumulated $P$ as a function of the incoming wave direction. 


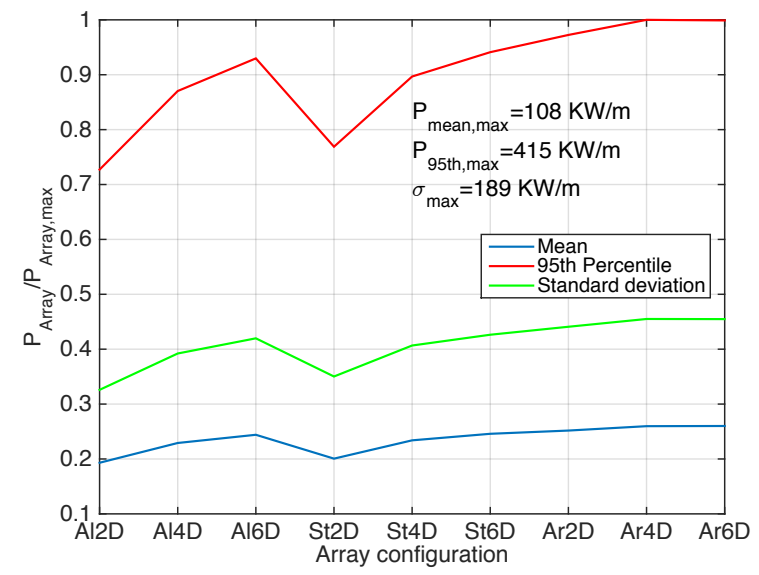

Figure 6: Mean (blue), 95th percentile (red) and standard deviation (green) of $P$ for the 9 array layouts during the life-cycle period between 1992 and 2016. 

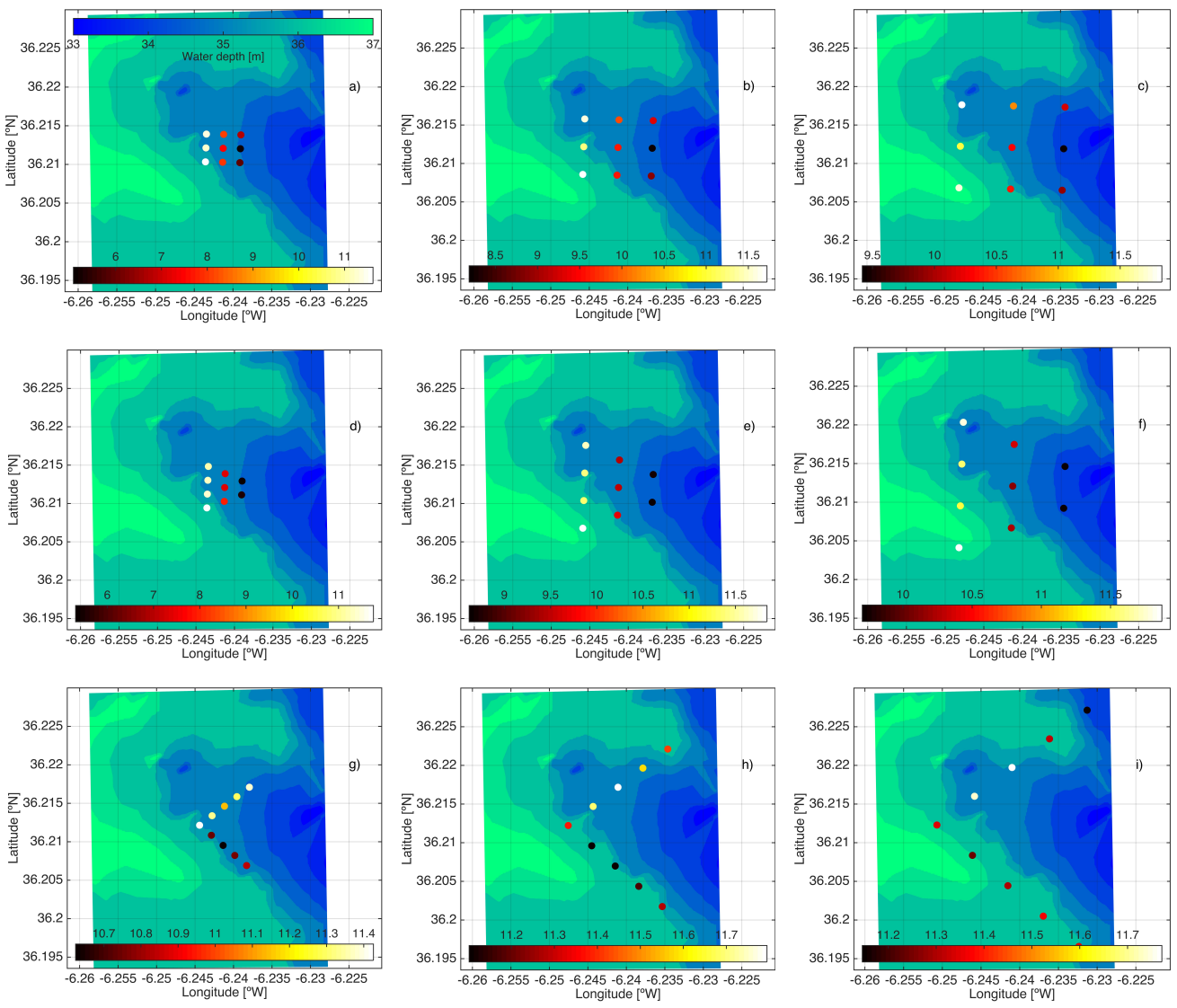

Figure 7: Mean $P$ in kW/m obtained for every WEC of: a-c) Aligned arrays, d-f) Staggered arrays, g-i) Arrow arrays. Blue-green colormap indicates water depths, black to white colormap indicates mean $P$. 


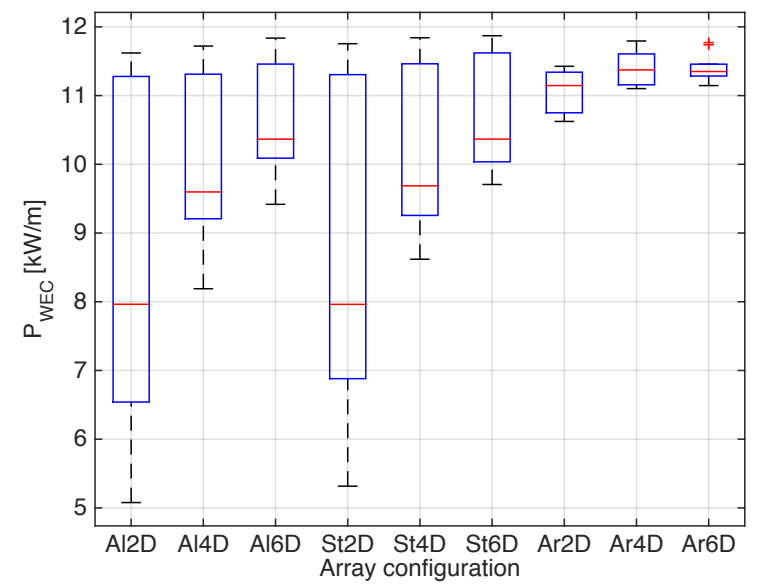

Figure 8: Statistical inference of the mean $P$ obtained for the WECs of the different array configurations. In the boxplot, the central marks indicate the median and the edges of the box the 25 th and 75 th percentiles. Black crosses represent the extreme values. 

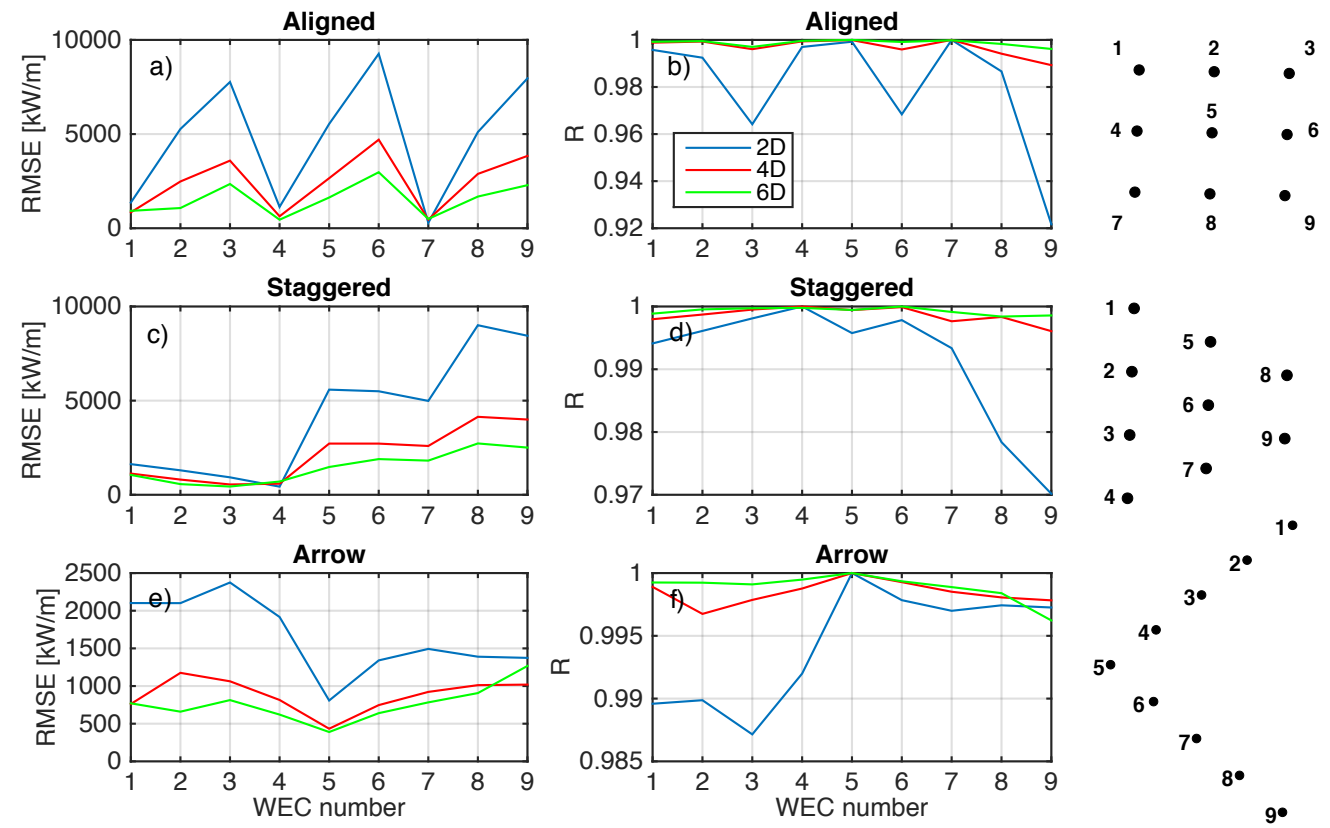

Figure 9: Root mean square error (RMSE) and correlation coefficient (R) between WECs in the arrays and an isolated WEC in the geometrical center of the array for the mean $P$ : a-b) Aligned, c-d) Staggered and e-f) Arrow. Schemes at the right of the figure represent the position of each WEC. 


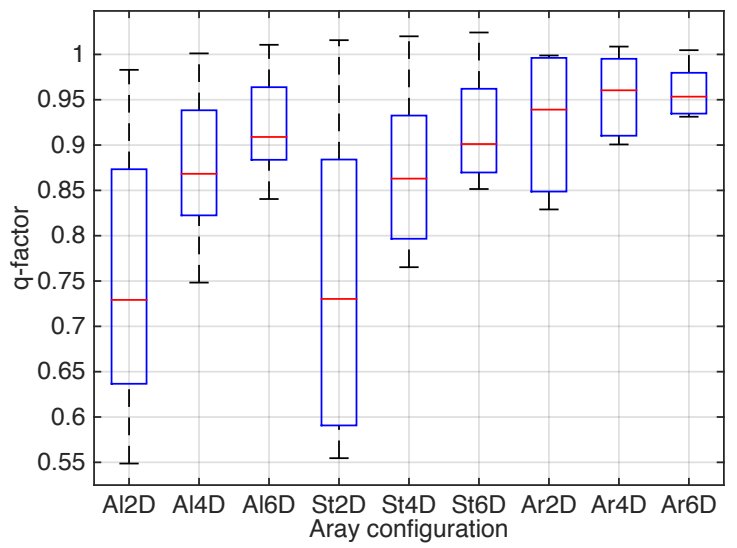

Figure 10: Statistical inference of the $q$-factor obtained for the WECs of each array configuration.
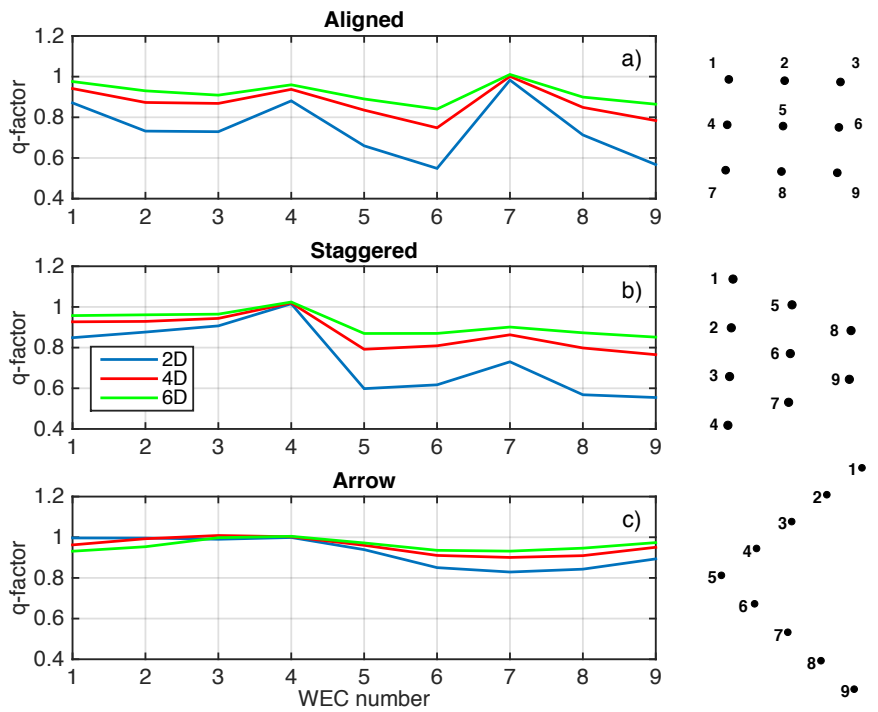

Figure 11: $q$-factor for the WECs locations for the 9 array layouts defined: Aligned (a), Staggered (b) and Arrow (c) geometries. Schemes in the right part of the figure represent the location of each WEC. 


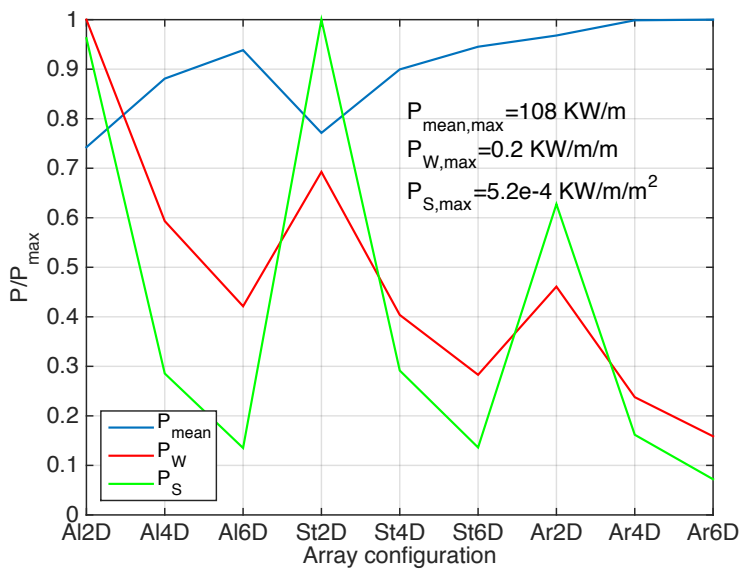

Figure 12: Non-dimensional mean $P$ (blue line), $P$ per unit of occupied wave front $\left(P_{W}\right.$, red line), $P$ per unit of occupied surface ( $P_{S}$, green line) obtained for the life-cycle period of the WECs between 1992 and 2016. 

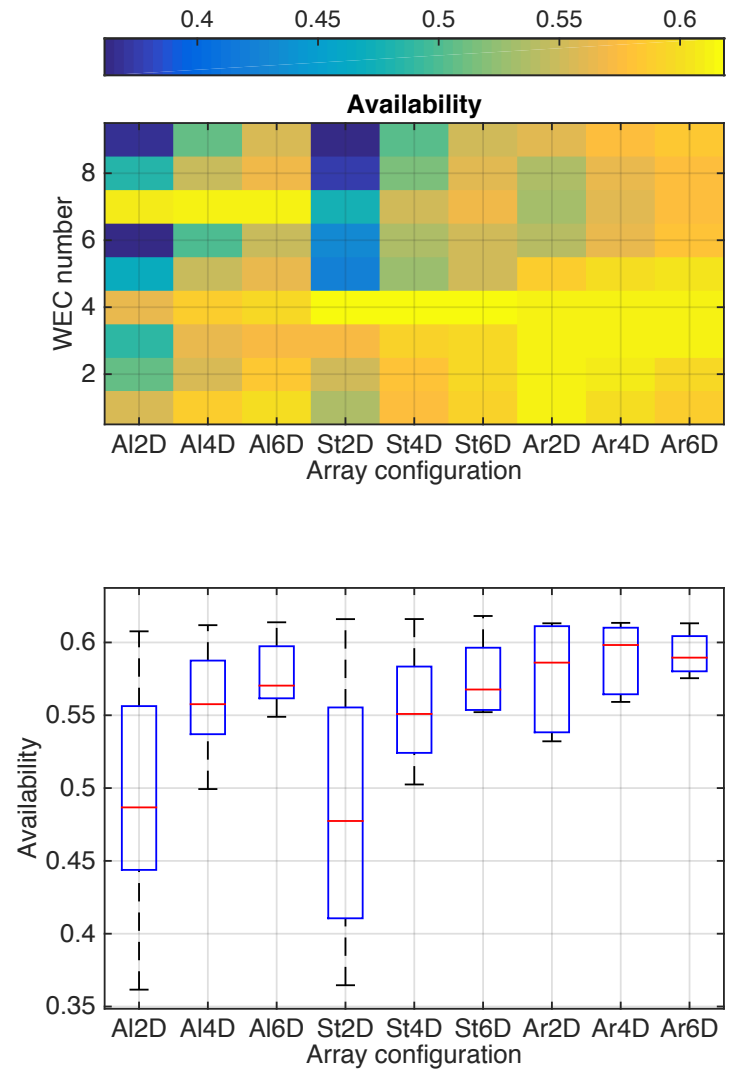

Figure 13: Availability (percentage of time during the life-cycle) obtained for the different WECs and array layouts. Upper panel depicts the mean availability for the life-cycle period for every WEC in each layout defined. Lower panel shows the statistical inference of these mean values. 

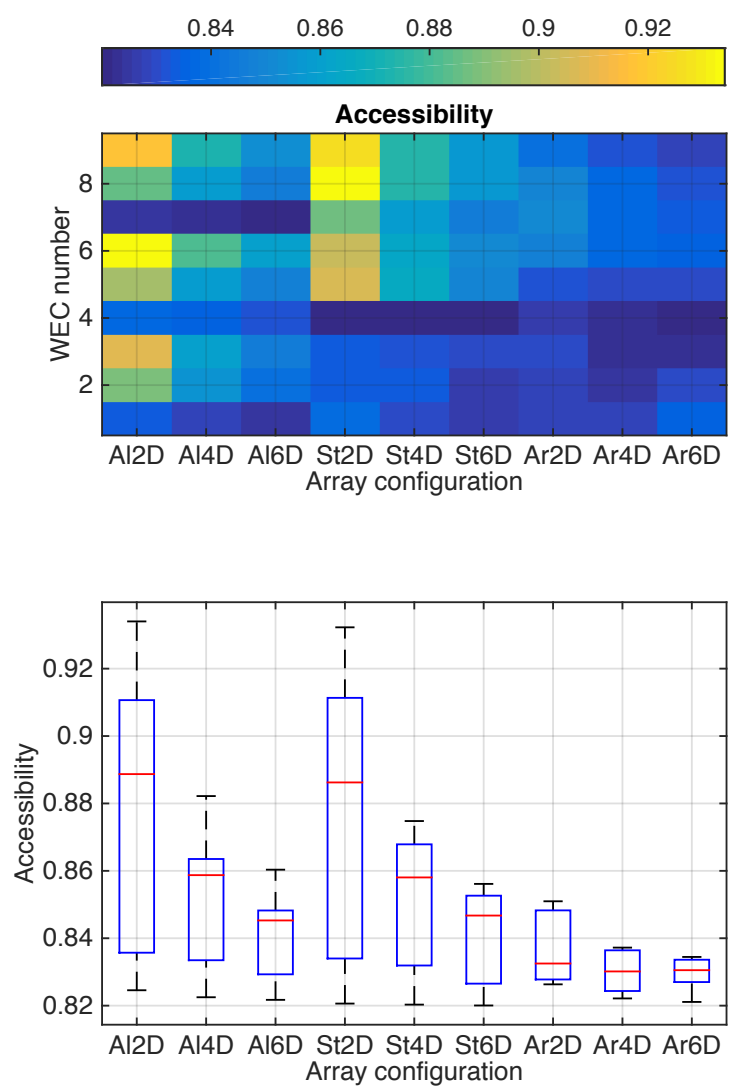

Figure 14: Accessibility obtained for the different WECs and array layouts. Upper panel depicts the mean accessibility for the life-cycle period for every WEC in each layout defined. Lower panel shows the statistical inference of these mean values. 

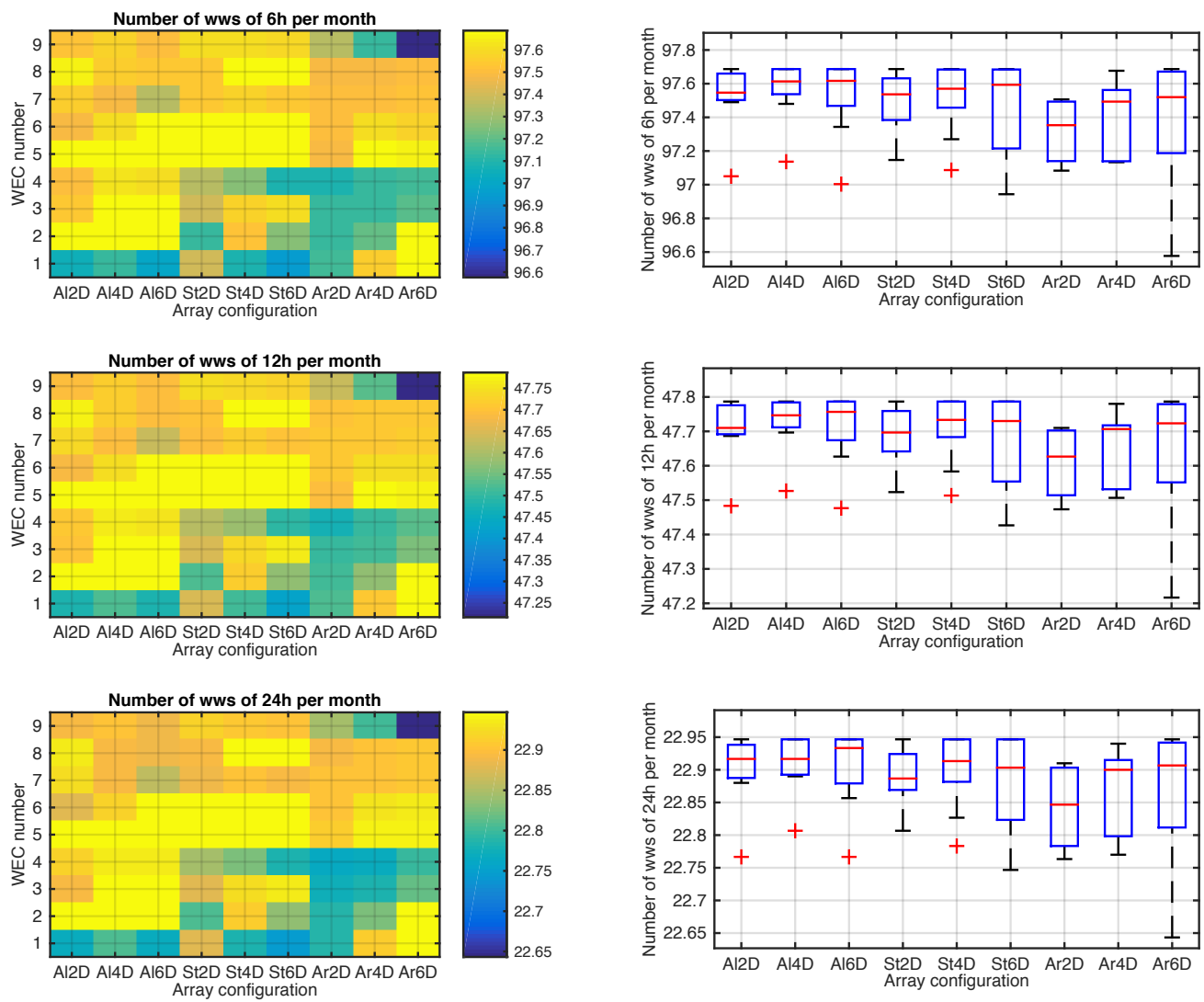

Figure 15: Mean monthly number of wws obtained for the different WECs and array layouts. Left panels depict the mean values for the life-cycle period for every WEC in each layout defined. Right panels show the statistical inference of these mean values. 

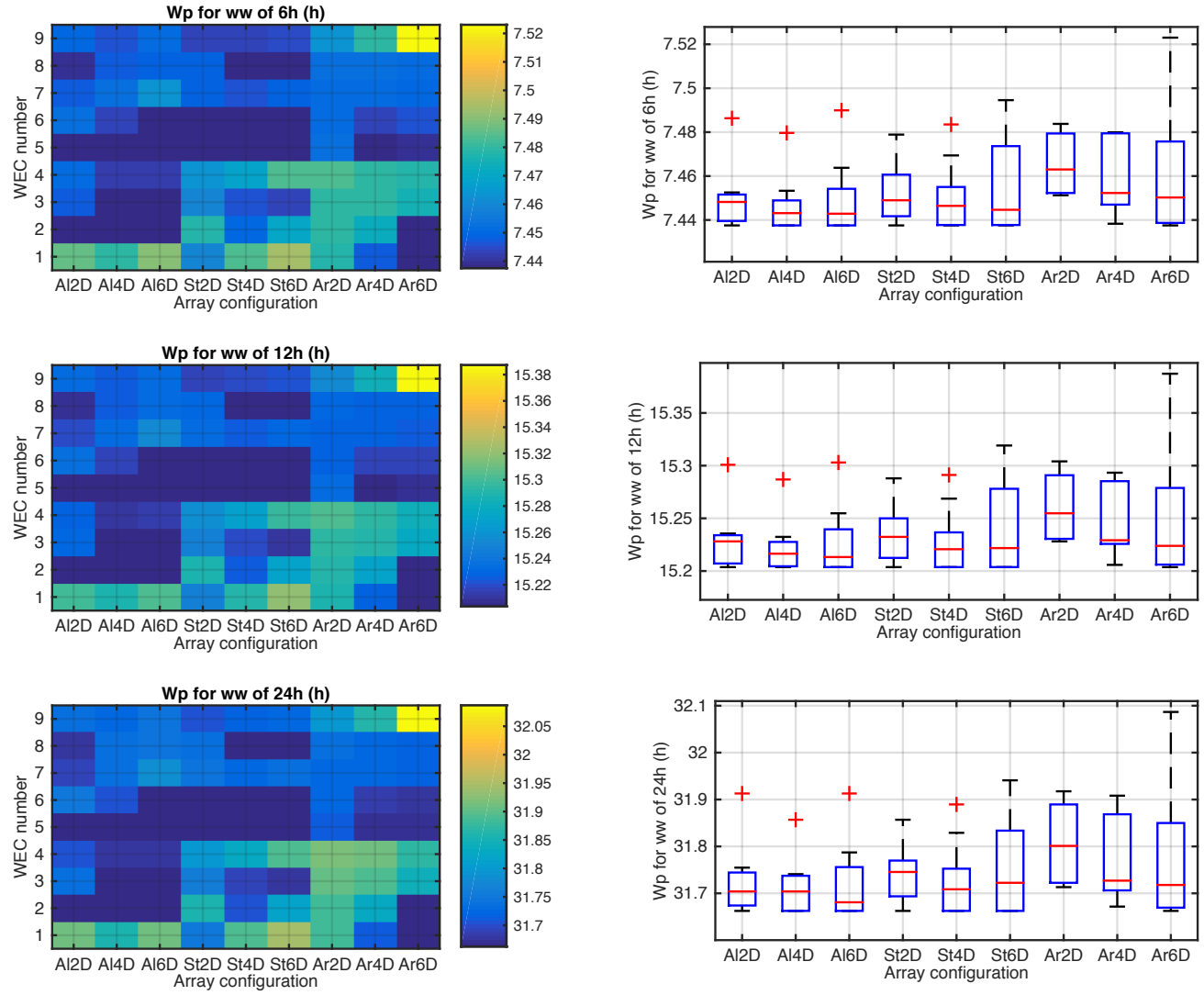

Figure 16: Mean wp obtained for 6,12 and $24 \mathrm{~h}$ wws for the different WECs and array layouts. Left panels depict the mean values for the life-cycle period for every WEC in each layout defined. Right panels show the statistical inference of these mean values. 